\title{
From Patient98 to Thor2005: from a Component-based Multimedia Workstation for Cardiac Care to a Complete Cardiology Information System
}

\author{
N van der Putten, S Nelwan, R Hamers, M de Wijs, F Smits, ML Simoons \\ Thoraxcentre, Erasmus MC, Rotterdam, The Netherlands
}

\begin{abstract}
A complete cardiology information system is under development. [1] This system should provide to physicians, nurses and others not only access to all patient data but also to the appropriate guideline information, as well as background material (journals, textbooks).

The different "building blocks" of the system have been developed or will be developed by different organisations.

The integration of the different blocks (systems) provides the physician access all to patient related information, for a specific difficult patient problem with the knowledge base related to his specific problem (what is the appropriate course of action) as well as registry/survey/information (what do my colleagues do in practice.

This article describes the following main characteristics of this system more extensively:

- Interactive guideline-based decision support

- General report generator

- Unique unambiguous relation between images, results and reports.
\end{abstract}

\section{Introduction}

The Thoraxcentre in Rotterdam is a joint institute of cardiology and cardiac surgery at the Erasmus Medical Centre.

We have already reported the development of Patient98, a custom built component-based medical workstation.[2] This workstation provides to physicians, nurses, and others access to all patient data including administrative data, summaries of studies and discharge letters, alpha numerical lab reports, signals (ECG etc), pictures (chest X-ray) and moving images (echocardiography, angiography). Patient98 is a modular patient-based system. The user interface reflects the traditional structure of the paper patient records: a folder with tabpages. Every tabpage represents one piece of diagnostic or therapeutic information.

Patient 98 is now in use for five years. At this moment about 1200 clinicians in the hospital have been registered as user. In the Thoraxcentre about 200 clinicians are using Patient98 in their daily practice.

The Patient98 system is expanded to a complete Cardiology Information System (Thor2005). Figure 1 illustrates the different sources of information and products (patient care, surveys, registries) of Thor2005 in an integrated manner.

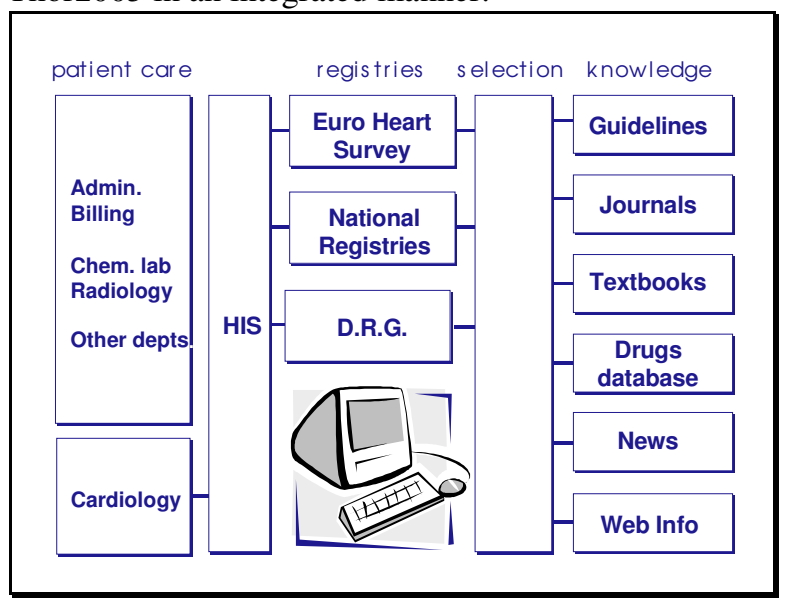

Figure 1. Main sources of information for Thor2005.

The main building blocks of the system are :

\section{a. Cardiology information system}

The information used by cardiologists for diagnosis and treatment of their patients varies from personal notes (history, physical examination), to signals and images (electrocardiograms, echocardiograms) reports from investigations and reports from procedures. In Thor2005 all this information can be provided in digital format. We are integrating: 
- Custom built cardiology subsystems (e.g. : echo result information system)

- Viewers for specific information systems of commercials vendors (e.g. for electrocardiography, diagnostic cardiac catheterisation and interventional cardiology

- Specific hospital-wide used subsystems for medication registration, report generation etc.

\section{b. The Hospital Information System (HIS)}

Cardiology is part of a larger hospital structure. The cardiology information system should be integrated with a full hospital information system. The latter may provide administrative information, billing services, access to the biochemistry- and other laboratories, access to radiology (reports and images), and exchange of information with other departments.

\section{c. Journals and textbooks}

The information in current textbooks and journals is available with the publishers in digital format. Much of this information is nowadays available on the web through libraries (e.g.: Science Direct, Ideal , other providers) or through personal subscriptions (Cardiosource etc.). The information is accessible through key words and other search systems at relatively low costs

We are developing in cooperation with Collexis and Elsevier a direct online access to textbooks and journals. The information is accessible through selected key words from our medical modules.

\section{d. Guidelines and educational material}

Guidelines for prevention, detection and management of cardiovascular disease are being developed through professional organisations such as the European Society of Cardiology. In addition many National Societies of cardiology develop their own guidelines, including for example the Netherlands. Again, much of this information is available at the web and can be searched through key words.

\section{e. Cardiology news}

News services, often sponsored by the pharmaceutical and device industry, but also provided by independent organisations such as the ESC, cover the major congresses, recent clinical trials etc.

Apart from the services mentioned above information on cardiovascular diseases, cardiology, cardiothoracic surgery and related topics can be found on many places on the web UpToDate, etc).

\section{$f$. Registries and surveys}

In some European countries, detailed information is collected systematically to assess the use of facilities and procedures (quality assurance) and/or the need for such (waiting lists).

Independently, the European Society of Cardiology has created the Euro Heart Survey Programme in order to verify the application of guidelines in clinical practice, verify the applicability of evidence based medicine (particularly clinical trials) and to assess the outcome of different patient management strategies. For similar reasons, registries and surveys are conducted by different national organisations throughout Europe. Most registries and survey require specific data collection and data entry, although already some are integrated in daily medical practice. For educational purposes, but also for patient specific decisions, it would be very useful if registry/survey information may be accessed by individual cardiologists.

In this paper we describe the following features of Thor2005 :

- Interactive guideline-based decision support

- General report generator

- Unique unambiguous relation between images, results and reports.

\section{Essential features}

\subsection{Interactive guideline-based decision support}

Clinical guidelines encapsulate the best medical practices, and are becoming ever more important in managing the explosion of medical knowledge and in promoting evidence-based medicine. A clinical guideline contains the intentions of the guideline, medical background, patient eligibility criteria, procedural statements such as clinical algorithms and drug recommendations, evidence for the advisories, treatment cost-benefit analysis, and references. The guidelines are generally published in textual format via print and electronic media.

In a project, launched by the ICIN, the Interuniversity Cardiology Institute of the Netherlands, all participating centers are implementing various (parts of) currently accepted national guidelines in a uniform way in Gaston a decision support system.

Gaston is a so called knowledge-base-centric 
system. It uses Protégé-2000, a knowledgeengineering environment, to facilitate patientspecific therapeutic advisories. In Rotterdam Gaston has been linked to Thor2005. We have implemented the guidelines for secondary prevention and diagnosis and treatment of Marfan patients. Domain experts have extracted information from the guideline text, have interpreted it, and then encoded it in a computable formalism in Gaston. We have connected Gaston to our systems for discharge and follow-up. Gaston uses patient data to interpret the knowledge base, generates a therapeutic advisory, and if required, justifies the advisory.

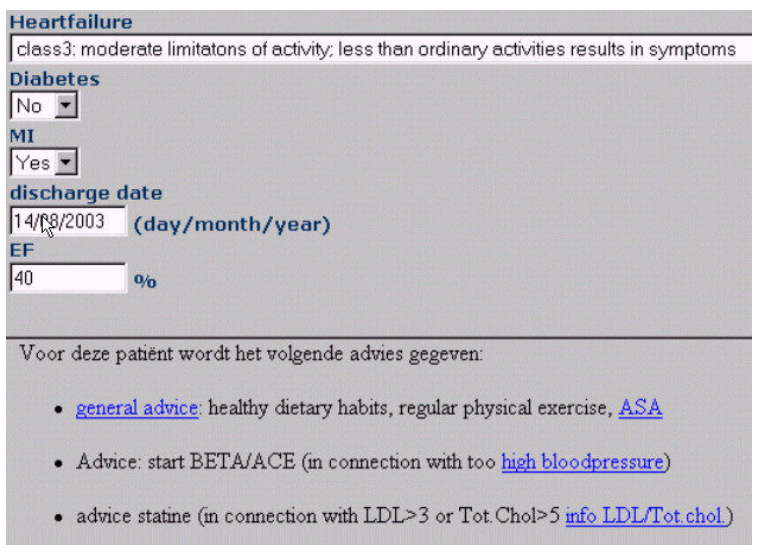

Figure 2. An example of secondary prevention advices in the discharge module.

Gaston generates an advice on the basis of the guidelines in the Gaston database, and the data on the discharge. The advice is only generated if Gaston has concluded that the cardiologist does not follow the guideline and the cardiologist had indicated that prefers to use Thor2005 in the advice generation mode.

The user may get more detailed relevant information of the presented guideline rule by clicking the rule.

We have developed a link mechanism between the guideline rules and the original guideline text in cooperation with Elsevier.

\begin{abstract}
2.2.3. Blood lipids
The blood cholesterol goals are a total cholesterol consistently below $5.0 \mathrm{mmol} / \mathrm{l}(190$ $\mathrm{mg} / \mathrm{dl})$, and an LDL-cholesterol below $3.0 \mathrm{mmol} / \mathrm{l}(115 \mathrm{mg} / \mathrm{dl})$. Concentrations of $\mathrm{HDL}-$ cholesterol and triglycerides are not used as goals of therapy. However, an HDLcholesterol $<1.0 \mathrm{mmol} / 1 / 40 \mathrm{mg} / \mathrm{dl})$ and fasting triglycerides $>2.0 \mathrm{mmol} / 1(180 \mathrm{mg} / \mathrm{dl})$ are markers of increased coronary risk. If the total and LDL-cholesterol goals are not achieved with lifestyle changes then drug therapy should be used. Preference should be given to HMG CO-A reductase inhibitors (statins) as this class of lipid lowering drugs has prenting coronary patients.
\end{abstract}

Figure 3. The supporting guideline text on blood lipids related to the third advice on the screen in figure 2 .

\subsection{General report generator}

Specific information systems have been developed for registration of patient specific data as collected during functional investigations, therapies, care in wards and intensive cares, follow ups and visits to the outpatient clinic. Every individual investigation, therapy, clinical stay, or out-patient clinic visit may result in a report. In case (nearly) all information, required for a report, can be provided in digital format, every report can be generated almost automatically. Some reports (e.g. for an ECG investigation) requires data from only one source. Other reports, especially an outpatient clinical or ward discharge report consists of information from different sources: patient history, physical examination, results and conclusions from (bio)chemical and instrumental examinations, conclusions extracted by all the analyzed data, and therapy suggestions etc. The Erasmus MC has developed a general report generator. This module facilitates the generation of reports making use of HIS data and information from the individual departmental systems. The interface between the report generator and other systems is based on $\mathrm{XML} / \mathrm{ODBC}$ and web services.

\subsection{Unique relation between images, results and reports}

For the clinical decision and follow up process a general review facility is expected to offer considerable value especially if the images and the related results and reports can be reviewed simultaneously. Patien98 facilitates the simultaneous presentation of the echocardiography images and the relating results on two screens. [3]

The contemporary cathlab is very often a collection of independent computerized systems for individual tasks such as hemodynamic analysis, digital angiography, quantitative coronary angiography (QCA) or ancillary imaging and quantitative analysis systems (e.g. intravascular ultrasound, IVUS, and quantitative coronary ultrasound, QCU). Only during the interventional catheter treatment many cathlabs have the opportunity to review angiography images and quantitative results simultaneously. After the intervention procedure the images and the results 
have been stored in different archives. Retrospective measurements, and simultaneous presentation of the results and the relating images are not facilitated. In the Thoraxcentre the QCA data are stored in CAASII files (PIE Medical), the angiograpy images in the Hemapacs (custom built development), the QCUdata in the Curad database and the IVUS images in the Curad PACS. Every QCA analysis results in two CAASII files : 1. A TIF-file, containing the analysed image and global analysis results 2. A TXT-file with detailed analysis results.

Curad (Wijk bij Duurstede, The Netherlands) has developed an integrated angio- and IVUS environment in co-operation with the Thoraxcentre. The Curad system can select and read angio cine runs from the Hemapacs (our angio Dicom server). A cine run can be held at a particular frame and dragged to a box compromising of (maximum 12) images to be used in subsequent QCA measurements. When all required frames are selected, the CAASII analysis routine can be launched for analysis. The Curad system constructs an image dataset from the result CAASII files (TIF + TXT) consisting of an image and Dicom header. This resulting Dicom file is sent to the Hemapacs, where the analysis results are imported in the database. This facilitates the hospitalwide simultaneous review of angio images and QCAresults.

The IVUS system has been described elsewhere. [4]

\section{Conclusion and discussion}

The design, and the current status of Patient 98 and its successor, Thor2005, have been described. Patient 98 is a general multimedia workstation for medical care with dedicated modules for cardiology and cardiac surgery. It integrates almost all medical patient information from a collection of different medical databases in one well-ordered, graphical interface. There is a wide acceptance of Patient 98 within the Erasmus Medical Centre of Rotterdam. Thor 2005 is an extension of Patient 98 by facilitating registration of personal notes during examination, diagnosis and therapy, simultaneous review of multimodality data, interactive guideline-based decision support, and online access to background material (books, articles, journals, guidelines). We like to emphasize that Thor 2005 is not a $100 \%$ custom built or a vendor specific system. It integrates information from different sources and different vendors in a standardized way. We envisage that the manufacturers of specific systems for echocardiography, angiography, cardiac intervention and electrophysiology etc. will continue to develop more sophisticated systems for their specific purposes. We will choose among vendors of different products, taking into account not only the system specifications and costs but also the options for integration.

\section{References}

[1] Simoons ML, van der Putten N, Wood D, Boersma E, Bassand JP. The Cardiology Information System: the need for data standards for integration of systems for patient care, registries and guidelines for clinical practice. In : Eur Heart J. 2002 Aug;23(15):1148-52.

[2] Putten $N$ van der, Nelwan S, Meij S, Smits F, Slingerland M, Suling R, Kroon F, Baljon M. Patient98: A Component Based Multimedia Workstation for Cardiac Care and Research. In: Computers in Cardiology 1999 ; IEEE Computer Society Press, 1999; 77-81.

[3] Putten, N van der, Hamers R, Nelwan S, Vletter W, Cate F ten, Dijk W, Baljon M. Desktop Echocardiography. In: Computers in Cardiology 2002; IEEE Computer Society Press, 2002; 341-344.

[4] Hamers R, Bruining N, Knook M, Sabate M, Roelandt JRTC. A Novel Approach to Quantitative Analysis of Intra Vascular Ultrasound Images. In: Computers in Cardiology 2001 ; IEEE Computer Society Press, 2001; 589-592.

Address for correspondence:

Niek van der Putten, PhD

Erasmus MC

Thoraxcentre

Dr. Molewaterplein 40

3015 GD Rotterdam

The Netherlands

E-mail: n.h.j.j.vanderputten@erasmusmc.nl 